\title{
Apolipoprotein A1 is associated with SYNT AX score in patients with a non-ST segment elevation myocardial infarction
}

\author{
Bang-Dang Chen ${ }^{1,2,3 \dagger}$, Xiao-Cui Chen ${ }^{1,2,3 \dagger}$, Yi-Ning Yang ${ }^{1,3,4}$, Xiao-Ming Gao ${ }^{1,2,3}$, Xiang Ma ${ }^{1,3,4}$, Ying Huang ${ }^{1,3,4}$, \\ Xiao-Mei Li $i^{1,3,4}$, Min-Tao Gai ${ }^{1,2,3}$, Fen Liu ${ }^{1,2,3}$, Shuo Pan ${ }^{5^{*}}$ (D) and Yi-Tong Ma ${ }^{1,2,3,4^{*}}$
}

\begin{abstract}
Background: The study was designed to investigate lipid profile and SYNTAX score in patients with non-ST segment elevation myocardial infarction (NSTEMI).

Methods: 311 patients with NSTEMI were enrolled. The demographic, clinical data, blood samples and SYNTAX score were documented. The Pearson linear correlation was used to detect confounding factors linearly correlated with SYNTAX score. The significantly correlated confounding factors were put into the multiple linear regressions.

Results: The Pearson linear correlation showed that high-density lipoprotein- cholesterol (HDL-C) and apolipoprotein A1 (ApoA1) were significantly correlated with Syntax Score $(r=-0.119, P=0.044$ and $r=-0.182, P=$ 0.002 , respectively). The multiple linear regressions for Syntax Score were built using HDL-C and ApoA 1 , respectively. After the adjustment of other significantly correlated confounding factors such as white blood cell count (WBC), myohemoglobin (MB), glutamic-oxalacetic transaminase (AST) and creatinine, the ApoA1 still showed significant association with Syntax Score $(\beta=-0.151, P=0.028)$. The area under curve was (AUC) 0.624 and the optimal cutoff value is $1.07 \mathrm{~g} / \mathrm{L}$ when using ApoA 1 to predict moderate and severe coronary artery lesions. The patients with ApoA $1 \geq 1.07 \mathrm{~g} / \mathrm{L}$ and $<1.07 \mathrm{~g} / \mathrm{L}$ have the Syntax Scores of $12.21 \pm 11.58$ and $16.33 \pm 11.53$, respectively $(P=0.001)$.

Conclusions: The ApoA1 is the only lipid factor significantly associated with complexity of coronary artery lesion in patients with NSTEMI, the patients with ApoA $1<1.07 \mathrm{~g} / \mathrm{L}$ may have more complex coronary artery lesions.
\end{abstract}

Keywords: Apolipoprotein A1, SYNTAX score, NSTEMI, Lipid

\section{Background}

Non-ST segment elevation myocardial infarction (NSTEMI) is one of the most severe and lethal form of myocardial infarction $[1,2]$. Although NSTEMI patients may have lower in-hospital mortality than patients with ST segment elevation myocardial infarction (STEMI), however, 4-year mortality in NSTEMI patients is twofold higher than that in patients with STEMI $[3,4]$.

The SYNTAX score, a lesion-based angiographic scoring system, has been widely used to grade the complexity of

\footnotetext{
* Correspondence: parker1985@sina.com; myt-xj@163.com

${ }^{+}$Bang-Dang Chen and Xiao-Cui Chen contributed equally to this work.

${ }^{5}$ First Department of Cardiology, People's Hospital of Shaanxi Province, Xi'an, China

${ }^{1}$ Clinical Medical Research Institute of First Affiliated Hospital of Xinjiang Medical University, Urumqi, China

Full list of author information is available at the end of the article
}

coronary artery disease. Several trials have showed that patients with a relatively high SYNTAX score have worse cardiovascular outcomes, and the score is an independent predictor of major adverse cardiovascular events (MACE) for percutaneous coronary intervention (PCI) [5-7]. The SYNTAX score of $\geq 33$ points is widely accepted criteria to select coronary artery bypass grafting (CABG) over PCI in treatment of the left main lesions. SYNTAX score of 22-32 points is defined as moderate complexity of coronary artery disease, both the CABG and PCI can be adopted [8].

Traditional lipid factors, such as total cholesterol (TC), triglycerides (TG), low-density lipoprotein cholesterol (LDL-C), high-density lipoprotein cholesterol (HDL-C) have been widely used as predictors in acute coronary syndrome $[9,10]$. Apolipoprotein A1 (ApoA1), the main protein component of HDL-C in the plasma has functions of

(C) The Author(s). 2019 Open Access This article is distributed under the terms of the Creative Commons Attribution 4.0 International License (http://creativecommons.org/licenses/by/4.0/), which permits unrestricted use, distribution, and 
protecting against atherosclerosis and cardiovascular disease, meanwhile it could also promote cellular cholesterol efflux and cholesterol transportation from peripheral tissues to the liver [11]. Apolipoprotein B (ApoB) is the main component of LDL-C, and its level could reflect the level of atherogenic lipoprotein particles [12, 13].

A previous study has investigated the association between the lipids factors and SYNTAX score in patients with stable coronary artery disease [14]. Since vast amount of NSTEMI patients had multi-vessel disease $[15,16]$, the evaluation of SYNTAX score is necessary and crucial to these patients in the selection of optimal treatments. The present study intended to investigate the association between lipids factors and SYNTAX score in NSTEMI patients.

\section{Methods}

\section{Subjects}

We performed this present cross-sectional and observational study in Cardiology Department of First Affiliated Hospital of Xinjiang Medical University from January of 2015 to June of 2018. The NSTEMI is diagnosed according to symptoms, electrocardiogram (ECG) and myocardial enzyme results. Symptoms of NSTEMI includes the chest pain, chest tightness or dyspnea, the durations should be over $30 \mathrm{~min}$. ECG diagnosis of NSTEMI is defined as: horizontal or downward ST segment depression $\geq 1 \mathrm{~mm}$ with upright, bidirectional or lightly inverted $\mathrm{T}$ wave, or symmetric and deep inversed $\mathrm{T}$-wave without obvious ST segment shift. Myocardial enzyme changes include significant elevation of creatine kinase (CK), creatine kinase-MB (CK-MB), lactate dehydrogenase $(\mathrm{LDH})$, Troponin I, myoglobin (MB), alanine aminotransferase (ALT), aspartate aminotransferase (AST), alkaline phosphatase (ALP) or transglutaminase according to features and occurrence time of each enzyme in NSTEMI patients. The patients who have symptoms, ECG and myocardial enzyme changes simultaneously are diagnosed as NSTEMI and could be enrolled in the present study. The main exclusion criteria include previous percutaneous stent implantation or percutaneous transluminal coronary angioplasty (PTCA) procedures, or previous lipid-lowering therapy and unwillingness to receive further coronary angiography (CAG) examination [17].

Three hundred eleven hospitalized NSTEMI patients were finally enrolled according to enrollment standard above, $212(68.2 \%)$ patients were male and 99 (31.8\%) patients were female. The average age was $61.80 \pm 28.08$ years.

\section{SYNTAX score}

All enrolled NSTEMI patients received the CAG examination, the SYNTAX score was calculated using SYNTAX score calculator (current calculator version: 2.28; http:// www.syntaxscore.com/calculator/start.htm) after the CAG procedure. Two experienced interventional cardiology physicians were in charge of calculating the SYNTAX scores. The third interventional cardiology physician should be involved in the calculation of SYNTAX scores when the scores were different. The NSTEMI patients with different SYNTAX scores were divided into two different groups, the mild lesion group (SYNTAX score $<22$ ) and moderate and severe lesion group (SYNTAX score $\geq 22$ ) [18].

\section{Clinical data and blood tests}

Demographic data and cardiovascular risk factors were obtained from the medical records. The peripheral blood was sampled for myocardial enzymes tests after the admission of the patients. Other peripheral blood was sampled from patients in a fasting state on the morning following the admission day. Venous blood samples were sent to Clinical Laboratory Department of First Affiliated Hospital of Xinjiang Medical University for red blood cells (RBC) counts, hemoglobin, platelet counts, white blood cells (WBC) counts, CK, CK-MB, LDH, Troponin I, MB, ALT, AST, ALP, transglutaminase, TC, triglyceride, HDL-C, LDLC, ApoA1, ApoB, urea nitrogen (BUN), creatinine, cystatin$\mathrm{C}$, total bilirubin (TBIL), direct bilirubin (DBIL), total protein (TP), albumin, D-Dimer (DD), glycosylated hemoglobin-A1c (HbA1c) and brain natriuretic peptide (BNP) detection using standard biochemical techniques [19].

\section{ECG and echocardiography}

ECG was performed after the admission of the patients using Nihon Kohden ECG machine (ECG1350P). The patients with horizontal or downwardly ST segment depression $\geq 1 \mathrm{~mm}$ with upright, bidirectional or lightly inverted $\mathrm{T}$ wave, or symmetric and deep inversed $\mathrm{T}$ wave without obvious ST segment shift were screened out for further enrollment.

The left ventricular ejection fraction (LVEF) was obtained using Doppler echocardiography conducted within 3 days of admission [20].

\section{Definition of risk factors}

Hypertension was defined as an average systolic blood pressure $\geq 140 \mathrm{mmHg}$, or an average diastolic blood pressure $\geq 90 \mathrm{mmHg}$, or both, or self-reported use of antihypertensive medication, or a self-reported history of hypertension.

Diabetes was defined as fasting plasma glucose $\geq 7.0$ $\mathrm{mmol} / \mathrm{L}$, or random plasma glucose $\geq 11.1 \mathrm{mmol} / \mathrm{L}$, or $2 \mathrm{~h}$ plasma glucose in oral glucose tolerance test $(\mathrm{OGTT}) \geq$ $11.1 \mathrm{mmol} / \mathrm{L}$, or use of insulin or oral hypoglycemic agents, or a self-reported history of diabetes. 


\section{Statistical analysis}

The statistical analysis was conducted using SPSS version 16.0 for Windows (SPSS Inc., Chicago, IL, USA). Continuous variables were expressed as mean \pm standard deviations and the differences between the mild lesions group and moderate and severe lesions group were analyzed using the Mann-Whitney U-test. Categorical variables were expressed as proportions and the differences in categorical variables were analyzed using chi-square test. Pearson correlation analysis was conducted to determine the correlation between SYNTAX score and each clinical and laboratory factor. The multiple linear regressions of SYNTAX score were performed using significantly correlated lipid factors, HDL-C and ApoA1, respectively. The receiver operating characteristic (ROC) curve of ApoA1 for the prediction of moderate and severe lesions was performed, then the AUC and cutoff points of ApoA1 to predict moderate and severe lesions were calculated. Additionally, the distance on the ROC curve of each ApoA1 was calculated as the square root of $\left[(1 \text {-sensitivity })^{2}+(1 \text { - specificity })^{2}\right]$. The ApoA1 value with the shortest distance on the ROC curve was considered as appropriate cutoff. Finally, the SYNTAX score between patients with different ApoA1 values were calculated and compared using Mann-Whitney U-test. Statistical significance was established at $P<0.05$.

\section{Results}

Baseline characteristics of NSTEMI patients with mild lesions or moderate and severe lesions were shown in Table 1. The percentage of male, WBC counts, troponin I, MB, BNP and SYNTAX score in NSTEMI patients with moderate and severe lesions were significantly higher than those in NSTEMI patients with mild lesions $(P<0.05)$. Meanwhile, the HDL-C, apolipoprotein A, albumin and LVEF in NSTEMI patients with moderate and severe lesions were significantly lower than those in NSTEMI patients with mild lesions $(P<0.05)$. The percentage of smoking, diabetes mellitus, hypertension and age, HR, SBP, DBP, RBC counts, hemoglobin, platelet counts, CK, CK-MB, LDH, ALT, AST, ALP, transglutaminase, TC, triglyceride, LDL-C, apolipoprotein B, BUN, creatinine, cystatin-C, TBIL, DBIL, TP, DD, HbA1c showed no significant difference between the NSTEMI patients with mild lesions or moderate and severe lesions.

Pearson correlation analysis between Syntax score and clinical and laboratory factors in NSTEMI patients was presented in Table 2. WBC counts, MB, AST and creatinine were significantly and positively correlated with SYNTAX score $(P<0.05)$. Meanwhile, HDL-C, apolipoprotein A and albumin were significantly and negatively correlated with SYNTAX score $(P<0.05)$. The age, HR, SBP, DBP, RBC counts, hemoglobin, platelet counts, CK,
CK-MB, LDH, Troponin I, ALT, ALP, transglutaminase, TC, triglyceride, LDL-C, apolipoprotein B, BUN, cystatin-C, TBIL, DBIL, TP, BNP, HbA1c, DD, LVEF showed no significant correlation with SYNTAX score.

Since the significantly correlated lipid factors, HDL-C and ApoA1 were highly resembled with each other, we have built the multiple linear regression model using HDL-C and ApoA1, respectively. Multiple linear regression of SYNTAX score using ApoA1 and other confounding factors in NSTEMI patients was shown in Table 3. After the adjustment of WBC, MB, AST, albumin and creatinine, which were the confounding factors linearly correlated with SYNTAX score, the ApoA1 was still significantly associated with SYNTAX score $(\beta=-$ $0.151, P=0.028$ ). Meanwhile, the multiple linear regression of SYNTAX score using HDL-C and other confounding factors in NSTEMI patients was shown in Table 4. After the adjustment of WBC, MB, AST, albumin and creatinine, the HDL-C was not significantly associated with SYNTAX score $(\beta=0.093, P=0.167)$.

The ROC curve of ApoA1 for the prediction of moderate and severe lesions in NSTEMI patients was presented in Fig. 1. The AUC of ApoA1 for the prediction of moderate and severe lesions was 0.624 (0.544-0.704). The cutoff points of ApoA1 to predict moderate and severe lesions were calculated in Table 5. The distances in ROC curve were calculated using various ApoA1 values, the shortest distance on the ROC curve was 0.579 and $1.07 \mathrm{~g} / \mathrm{L}$ of ApoA1 was considered as the optimal cutoff in the prediction of moderate and severe lesions in NSTEMI patients with the sensitivity of 0.631 and specificity of 0.554 .

The SYNTAX score between patients with ApoA1 $\geq$ 1.07 and ApoA $1<1.07$ were presented in Fig. 2 . The patients with ApoA $1<1.07$ had significantly higher SYNT AX score $(16.33 \pm 11.53 \mathrm{~g} / \mathrm{L})$ than the patients with ApoA1 $\geq 1.07(12.21 \pm 11.58 \mathrm{~g} / \mathrm{L})$.

\section{Discussion}

Previous studies have reported that up to $80 \%$ of NSTEMI patient had multi-vessel disease, the complexity of coronary artery disease in NSTEMI patients was much higher than those in STEMI patients $[16,21]$. The appliance of the SYNTAX score was crucial since it reflected the complexity of coronary artery lesions in NSTEMI patients with more complicated coronary artery lesions. Meanwhile, Garg et al. [22] demonstrated that the SYNTAX score has the power to predict major adverse cardiovascular events and mortality. Other studies have also reported that the adverse long-term cardiovascular events showed significant increase as the SYNT AX score increased [23-25]. The SYNTAX score is an effective tool to affect the decision-making between CABG and PCI [26]. If the SYNTAX score was $\geq 33$ 
Table 1 Baseline characteristics of NSTEMI patients with mild lesions or moderate and severe lesions

\begin{tabular}{|c|c|c|c|}
\hline & Mild lesion (SYNTAX score $<22 ; n=242$ ) & Moderate and severe lesion (SYNTAX score $\geq 22 ; n=69$ ) & $P$ value \\
\hline Male (\%) & $154(63.6 \%)$ & $58(84.1 \%)$ & $0.001^{*}$ \\
\hline Smoking (\%) & $70(28.9 \%)$ & $25(36.2 \%)$ & 0.245 \\
\hline Diabetes mellitus (\%) & $43(17.8 \%)$ & $13(18.8 \%)$ & 0.838 \\
\hline Hypertension (\%) & $121(50.0 \%)$ & $33(47.8 \%)$ & 0.750 \\
\hline Age (years) & $61.63 \pm 31.67$ & $62.78 \pm 8.91$ & 0.076 \\
\hline HR (beat/min) & $73.04 \pm 13.57$ & $74.57 \pm 16.06$ & 0.477 \\
\hline SBP $(\mathrm{mmHg})$ & $129.21 \pm 18.59$ & $130.60 \pm 20.39$ & 0.796 \\
\hline $\mathrm{DBP}(\mathrm{mmHg})$ & $78.53 \pm 11.33$ & $79.53 \pm 12.14$ & 0.843 \\
\hline RBC counts $\left(\times 10^{12} / \mathrm{L}\right)$ & $4.54 \pm 1.03$ & $4.42 \pm 0.53$ & 0.830 \\
\hline Hemoglobin (g/L) & $137.17 \pm 16.49$ & $138.24 \pm 14.50$ & 0.493 \\
\hline Platelet counts $\left(\times 10^{9} / \mathrm{L}\right)$ & $198.43 \pm 64.01$ & $197.26 \pm 62.67$ & 0.852 \\
\hline WBC counts $\left(\times 10^{9} / \mathrm{L}\right)$ & $7.27 \pm 2.47$ & $8.38 \pm 2.82$ & $0.005^{*}$ \\
\hline CK (U/L) & $349.20 \pm 740.63$ & $371.30 \pm 735.81$ & 0.223 \\
\hline CK-MB (U/L) & $33.04 \pm 51.47$ & $37.59 \pm 63.13$ & 0.322 \\
\hline LDH (U/L) & $300.19 \pm 248.24$ & $284.07 \pm 204.26$ & 0.587 \\
\hline Troponin I (ng/mL) & $4.03 \pm 12.16$ & $16.67 \pm 100.61$ & $0.001^{*}$ \\
\hline $\mathrm{MB}(\mathrm{ng} / \mathrm{mL})$ & $75.84 \pm 180.60$ & $132.54 \pm 353.36$ & $0.004^{*}$ \\
\hline $\operatorname{ALT}(U / L)$ & $32.96 \pm 27.62$ & $34.70 \pm 31.67$ & 0.678 \\
\hline AST (U/L) & $50.13 \pm 70.06$ & $66.75 \pm 122.93$ & 0.449 \\
\hline ALP (U/L) & $95.16 \pm 29.19$ & $91.97 \pm 28.21$ & 0.756 \\
\hline Transglutaminase (U/L) & $35.41 \pm 49.02$ & $36.68 \pm 35.24$ & 0.937 \\
\hline TC (mmol/L) & $4.03 \pm 1.06$ & $3.92 \pm 1.00$ & 0.519 \\
\hline Triglyceride (mmol/L) & $1.74 \pm 1.52$ & $1.62 \pm 0.71$ & 0.514 \\
\hline $\mathrm{HDL}-\mathrm{C}(\mathrm{mmol} / \mathrm{L})$ & $1.08 \pm 0.75$ & $0.97 \pm 0.22$ & $0.001^{*}$ \\
\hline LDL-C (mmol/L) & $2.21 \pm 0.75$ & $2.33 \pm 0.77$ & 0.227 \\
\hline Apolipoprotein A1 (g/L) & $1.14 \pm 0.19$ & $1.06 \pm 0.19$ & $0.002^{*}$ \\
\hline Apolipoprotein B (g/L) & $0.84 \pm 0.22$ & $0.88 \pm 0.22$ & 0.154 \\
\hline BUN (mmol/L) & $5.16 \pm 1.63$ & $5.29 \pm 2.16$ & 0.898 \\
\hline Creatinine (umol/L) & $75.75 \pm 18.23$ & $82.32 \pm 26.27$ & 0.117 \\
\hline Cystatin-C (mg/L) & $1.50 \pm 7.02$ & $1.12 \pm 0.40$ & 0.514 \\
\hline TBIL (umol/L) & $16.23 \pm 9.97$ & $16.52 \pm 7.84$ & 0.609 \\
\hline DBIL (umol/L) & $5.30 \pm 2.49$ & $5.87 \pm 3.24$ & 0.163 \\
\hline TP (g/L) & $64.16 \pm 6.83$ & $62.36 \pm 5.27$ & 0.067 \\
\hline Albumin (g/L) & $38.75 \pm 3.99$ & $37.57 \pm 3.59$ & $0.018^{*}$ \\
\hline $\mathrm{DD}(\mathrm{ug} / \mathrm{mL})$ & $0.30 \pm 0.58$ & $0.33 \pm 0.50$ & 0.451 \\
\hline HbA1c (\%) & $6.25 \pm 1.50$ & $6.36 \pm 1.19$ & 0.350 \\
\hline BNP (pg/mL) & $246.57 \pm 520.58$ & $353.81 \pm 368.23$ & $<0.001^{*}$ \\
\hline LVEF (\%) & $58.90 \pm 8.90$ & $54.92 \pm 8.83$ & $0.001^{*}$ \\
\hline SYNTAX score & $8.61 \pm 7.09$ & $30.87 \pm 7.96$ & $<0.001^{*}$ \\
\hline
\end{tabular}

Note: NSTEMI Non-ST-Elevation Myocardial Infarction, HR Heart Rate, SBP Systolic Blood Pressure, DBP Diastolic Blood Pressure, RBC Red Blood Cells, WBC White Blood Cells, CK Creatine Kinase, CK-MB Creatine Kinase-MB, LDH Lactate DeHydrogenase, MB MyogloBin, ALT ALanine aminoTransferase, AST ASpartate aminoTransferase, ALP ALkaline Phosphatase, TC Total Cholesterol, HDL-C High-Density Lipoprotein-Cholesterol, LDL-C Low-Density Lipoprotein-Cholesterol, BUN Urea Nitrogen, TBIL Total Bilirubin, DBIL Direct Bilirubin, TP Total Protein, BNP Brain Natriuretic Peptide, HbA1c Glycosylated Hemoglobin-A1c, DD D-Dimer, LVEF Left ventricular ejection fraction, ${ }^{*} P<0.05$ 
Table 2 Pearson correlation analysis between SYNTAX score and clinical and laboratory factors in NSTEMI patients

\begin{tabular}{|c|c|c|}
\hline & $r$ & $P$ value \\
\hline Age & 0.034 & 0.545 \\
\hline$H R$ & 0.068 & 0.235 \\
\hline SBP & 0.031 & 0.583 \\
\hline DBP & 0.026 & 0.650 \\
\hline RBC counts & -0.018 & 0.750 \\
\hline Hemoglobin & -0.002 & 0.965 \\
\hline Platelet counts & -0.015 & 0.795 \\
\hline WBC counts & 0.221 & $<0.001^{*}$ \\
\hline CK & 0.062 & 0.363 \\
\hline CK-MB & 0.105 & 0.113 \\
\hline LDH & 0.059 & 0.393 \\
\hline Troponin I & 0.085 & 0.184 \\
\hline MB & 0.155 & $0.015^{*}$ \\
\hline ALT & 0.012 & 0.833 \\
\hline AST & 0.184 & $0.001^{*}$ \\
\hline ALP & -0.074 & 0.238 \\
\hline Transglutaminase & 0.006 & 0.922 \\
\hline TC & 0.020 & 0.740 \\
\hline Triglyceride & -0.049 & 0.408 \\
\hline $\mathrm{HDL}-\mathrm{C}$ & -0.119 & $0.044^{*}$ \\
\hline LDL-C & 0.077 & 0.196 \\
\hline Apolipoprotein A1 & -0.182 & $0.002^{*}$ \\
\hline Apolipoprotein B & 0.081 & 0.171 \\
\hline BUN & -0.004 & 0.941 \\
\hline Creatinine & 0.136 & $0.019^{*}$ \\
\hline Cystatin-C & 0.045 & 0.442 \\
\hline TBIL & 0.058 & 0.316 \\
\hline DBIL & 0.084 & 0.147 \\
\hline TP & -0.094 & 0.103 \\
\hline Albumin & -0.154 & $0.008^{*}$ \\
\hline BNP & -0.021 & 0.744 \\
\hline $\mathrm{HbA1c}$ & 0.117 & 0.079 \\
\hline DD & 0.090 & 0.763 \\
\hline LVEF & -0.082 & 0.220 \\
\hline
\end{tabular}

Note: NSTEMI Non-ST-Elevation Myocardial Infarction, HR Heart Rate, SBP Systolic Blood Pressure, DBP Diastolic Blood Pressure, RBC Red Blood Cells, WBC White Blood Cells, CK Creatine Kinase, CK-MB Creatine Kinase-MB, LDH Lactate DeHydrogenase, MB MyogloBin, ALT ALanine aminoTransferase, AST ASpartate aminoTransferase, ALP ALkaline Phosphatase, TC Total Cholesterol, HDL-C High-Density Lipoprotein-Cholesterol, LDL-C Low-Density LipoproteinCholesterol, BUN Urea Nitrogen, TBIL Total Bilirubin, DBIL Direct Bilirubin, TP Total Protein, BNP Brain Natriuretic Peptide, HbA1c Glycosylated HemoglobinA1c, DD D-Dimer, LVEF Left ventricular ejection fraction, ${ }^{*} P<0.05$

points in the left main lesion, the CABG should be considered as the priority treatment rather than PCI. In this present study, 242 patients (77.8\%) had SYNTAX score < 22 points and 69 patients $(22.2 \%)$ had SYNTAX score $\geq$
Table 3 Multiple linear regression of SYNTAX score using ApoA1 and other confounding factors in NSTEMI patients

\begin{tabular}{|c|c|c|c|c|}
\hline & \multicolumn{2}{|c|}{$\begin{array}{l}\text { Unstandardized } \\
\text { coefficients }\end{array}$} & \multirow{2}{*}{$\begin{array}{l}\text { Standardized } \\
\text { coefficients } \\
\beta\end{array}$} & \multirow[t]{2}{*}{$P$ value } \\
\hline & $B$ & S.E. & & \\
\hline WBC & 0.427 & 0.339 & 0.091 & 0.220 \\
\hline $\mathrm{MB}$ & 0.003 & 0.004 & 0.066 & 0.403 \\
\hline AST & 0.008 & 0.010 & 0.065 & 0.432 \\
\hline Albumin & -0.181 & 0.199 & -0.063 & 0.364 \\
\hline Creatinine & 0.056 & 0.041 & 0.093 & 0.174 \\
\hline ApoA1 & -9.270 & 4.194 & -0.151 & $0.028^{*}$ \\
\hline
\end{tabular}

Note: NSTEMI Non-ST-Elevation Myocardial Infarction, ApoA1 Apolipoprotein A1, WBC White Blood Cell, MB Myoglobin, AST Aspartate Transaminase

22 points, the patients with moderate and severe lesions had mean \pm S.D. SYNTAX score of $30.87 \pm 7.96$ points.

Among the documented lipid factors, the present study showed that HDL-C and ApoA1 were linearly and significantly correlated with SYNTAX score. Since the HDL-C and ApoA1 were highly resembled with each other, we have built the multiple linear regression models using HDL-C and ApoA1, respectively. After the adjustment of WBC, MB, AST, albumin and creatinine, the ApoA1 was still significantly associated with SYNT AX score $(\beta=-0.151, P=0.028)$. However, after the adjustment of WBC, MB, AST, albumin and creatinine, the HDL-C was not significantly associated with SYNTAX score $(\beta=0.093, P=0.167)$. The mechanism of the association between the ApoA1 and SYNTAX score in NSTEMI patients is not entirely clear. The possible mechanism may be as follows, human or recombinant ApoA1 has been shown to increase HDL-C efflux capacity and to reduce atherosclerotic disease. Several studies have identified ApoA1 as a key determinant of macrophage cholesterol efflux capacity, ApoA1 proteins may reduce atherosclerosis via regulating cholesterol efflux from macrophages $[9,27]$. Another study has made a reconstituted infusible human ApoA1, CSL112, the

Table 4 Multiple linear regression of SYNTAX score using HDL-C and other confounding factors in NSTEMI patients

\begin{tabular}{|c|c|c|c|c|}
\hline & \multicolumn{2}{|c|}{$\begin{array}{l}\text { Unstandardized } \\
\text { coefficients }\end{array}$} & \multirow{2}{*}{$\begin{array}{l}\text { Standardized } \\
\text { coefficients } \\
\beta\end{array}$} & \multirow[t]{2}{*}{$P$ value } \\
\hline & $B$ & S.E. & & \\
\hline WBC & 0.470 & 0.340 & 0.103 & 0.169 \\
\hline MB & 0.003 & 0.004 & 0.068 & 0.396 \\
\hline AST & 0.009 & 0.010 & 0.073 & 0.385 \\
\hline Albumin & -0.240 & 0.198 & -0.083 & 0.226 \\
\hline Creatinine & 0.060 & 0.042 & 0.099 & 0.153 \\
\hline HDL-C & -4.308 & 3.105 & 0.093 & 0.167 \\
\hline
\end{tabular}

Note: NSTEMI Non-ST-Elevation Myocardial Infarction, HDL-C High Density Lipoprotein Cholesterol, WBC White Blood Cell, MB Myoglobin, AST Aspartate Transaminase 


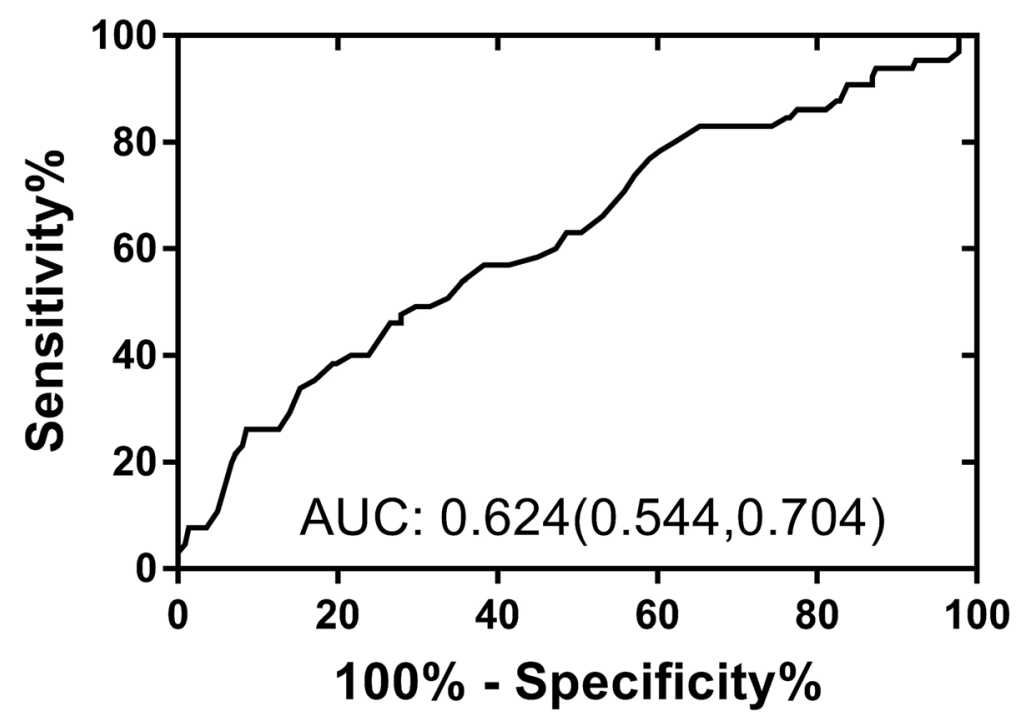

Fig. 1 The ROC curve of ApoA1 for the prediction of moderate and severe lesions in NSTEMI patients

CSL112 were injected to ACS patients and patients with stable atherosclerotic disease, the function of acute cholesterol efflux enhancement was confirmed in both population, and the benefit of CSL112 to reduce major adverse cardiovascular events remained to be seen in the following study $[11,28]$.

The study also demonstrated that The AUC of ApoA1 for the prediction of moderate and severe lesions was 0.624 (0.544-0.704) and $1.07 \mathrm{~g} / \mathrm{L}$ of ApoA1 was considered as the optimal cutoff in the prediction of moderate

Table 5 Sensitivity, specificity, and distance in the ROC curve of ApoA1 for the prediction of moderate and severe coronary artery lesions in NSTEMI patients

\begin{tabular}{llll}
\hline ApoA1 cutoffs $(\mathrm{g} / \mathrm{L})$ & Sensitivity & Specificity & Distance in ROC curve \\
\hline 0.75 & 1.000 & 0.000 & 1.000 \\
0.80 & 0.996 & 0.039 & 0.961 \\
0.90 & 0.910 & 0.262 & 0.744 \\
1.00 & 0.748 & 0.431 & 0.622 \\
1.06 & 0.653 & 0.523 & 0.590 \\
1.07 & 0.631 & 0.554 & 0.579 \\
1.08 & 0.602 & 0.569 & 0.587 \\
1.09 & 0.568 & 0.577 & 0.605 \\
1.10 & 0.539 & 0.593 & 0.615 \\
1.11 & 0.521 & 0.616 & 0.614 \\
1.20 & 0.334 & 0.831 & 0.687 \\
1.30 & 0.200 & 0.862 & 0.812 \\
1.40 & 0.093 & 0.938 & 0.910 \\
1.50 & 0.041 & 0.954 & 0.960 \\
1.85 & 0.000 & 1.000 & 1.000 \\
\hline
\end{tabular}

Note: NSTEMI Non-ST-Elevation Myocardial Infarction, ROC Receiver operating characteristic curve, ApoA1 Apolipoprotein A1 and severe lesions in NSTEMI patients with the sensitivity of 0.631 and specificity of 0.554 . The patients with ApoA $1<1.07$ had significantly higher SYNTAX score $(16.33 \pm 11.53 \mathrm{~g} / \mathrm{L})$ than the patients with ApoA1 $\geq 1.07$ $(12.21 \pm 11.58 \mathrm{~g} / \mathrm{L})$. The $1.07 \mathrm{~g} / \mathrm{L}$ of ApoA1 was selected as the optimal cutoff in the prediction of moderate and severe lesions, the NSTEMI patients with ApoA $1<1.07 \mathrm{~g} / \mathrm{L}$ may have more severe coronary artery lesions. The establishment the cutoff values added the clinical use to possible coronary artery lesions, the physicians should be alerted when the ApoA1 was $<1.07 \mathrm{~g} / \mathrm{L}$ since multi-vessel or severe coronary artery lesions may lead to worse clinical outcomes.

Aksakal et al. [29] reported that the presence of diabetes mellitus, no previous statin use, lower values of HDL-C, LVEF, estimated glomerular filtration rate were independent predictors of coronary artery complexity assessed using the SYNTAX score. This study has confirmed the HDL-C and creatinine were linearly correlated with coronary artery complexity. Meanwhile, the LVEF was significantly different between the patients with mild lesion and patients with moderate and severe lesion. However, the presence of diabetes mellitus and HbA1c showed no relationship with the SYNTAX score, the difference may come from the enrollment of patients, our patients were NSTEMI patients while the majority of patients from Aksakal et al. [29] were stable coronary artery disease patients. The study also documented the MB and AST were linearly correlated with SYNTAX score, the level of MB and AST may reflect the myocardial injury in NSTEMI patients, the level of myocardial injury may reflect the complexity and severity of coronary artery lesions determined by SYNTAX score [30]. 


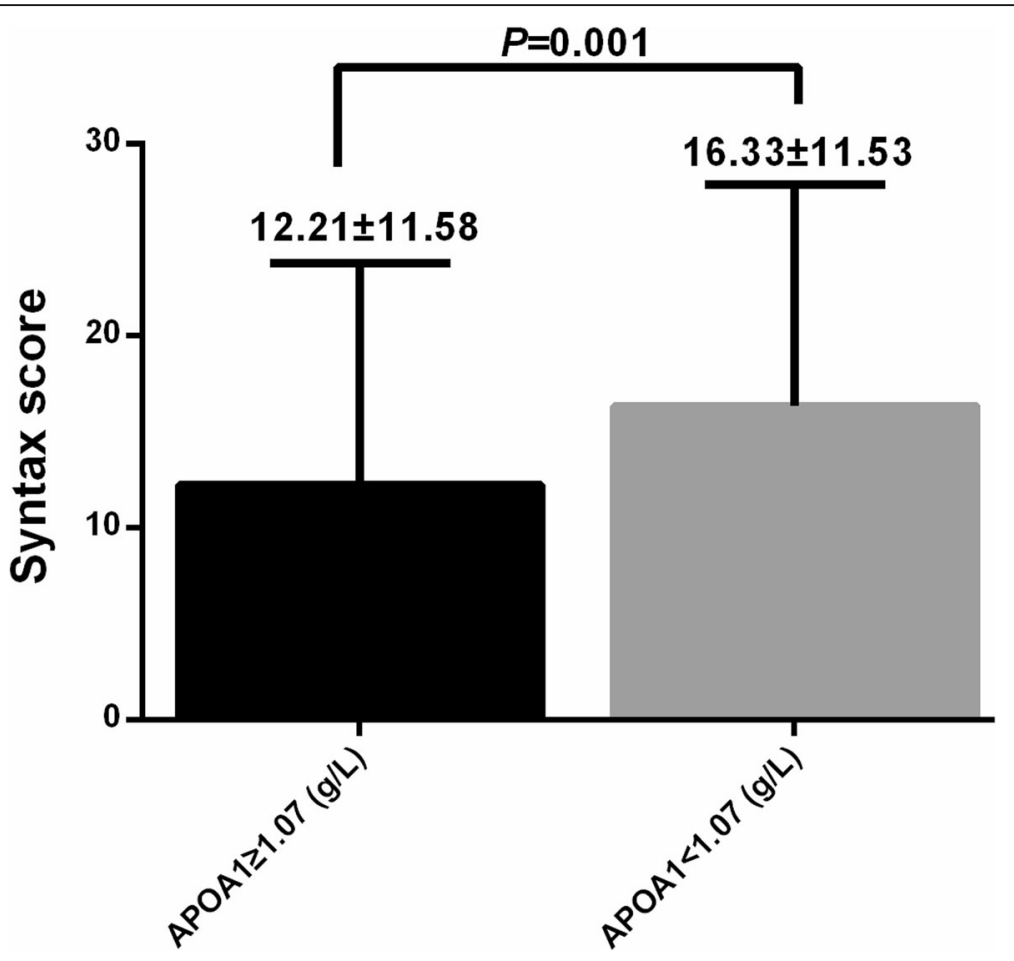

Fig. 2 The SYNTAX score between patients with ApoA1 $\geq 1.07$ and ApoA $1<1.07$

The strengths of the study are that it is the first association study between the lipid factors and complexity of coronary artery lesions in NSTEMI patients. Second, the study enrolled the cardiac enzymes and myocardial damage marker such as CK, CK-MB, LDH, Troponin I, MB, ALT, AST, ALP and transglutaminase, the significant different markers were adjusted as confounding factors. Third, the study excluded previous percutaneous stent implantation or percutaneous transluminal coronary angioplasty (PTCA) procedures, the SYNTAX score may precisely reflect the original status in NSTEMI patients, the study also excluded the patients with previous lipidlowering therapy, which guaranteed the accuracy of original lipids status. The study has several limitations, it is an cross-section study that it could not determine the causal relationship between the ApoA1 and SYNTAX score in NSTEMI patients. Second, the significantly different distributed gender was not in the linear regression since it is a categorical, the limited women number made it difficult for subgroup analysis. Third, we have observed that the $P$ value for HDL-C was 0.167 , meanwhile, the LDL-C and Apo B were also not correlated with SYNTAX score, the significance may change if the more NSTEMI patients were enrolled.

\section{Conclusion}

In conclusion, the ApoA1 is associated with SYNTAX score in NSTEMI patients. $1.07 \mathrm{~g} / \mathrm{L}$ of ApoA1 was considered as the optimal cutoff in the prediction of moderate and severe lesions in NSTEMI patients with the sensitivity of 0.631 and specificity of 0.554 . The further study may need larger sample size to further detect the association HDL-C, LDL-C, Apo B and SYNTAX score in NSTEMI patients, meanwhile, the underlying mechanisms in both animal and human models, and the effectiveness and safety of supplementary therapy in human still needed further exploration.

\section{Abbreviations}

ALP: alkaline phosphatase; ALT: alanine aminotransferase;

ApoA1: apolipoprotein A1; ApoB: Apolipoprotein B; AST: aspartate aminotransferase; AUC: area under curve; BNP: brain natriuretic peptide; BUN: urea nitrogen;" CABG: coronary artery bypass grafting; CAG: coronary angiography; CK: creatine kinase; CK-MB: creatine kinase-MB; DBIL: direct bilirubin; DD: D-Dimer; ECG: electrocardiogram; HbA1c: glycosylated hemoglobin-A1c; HDL-C: high-density lipoprotein- cholesterol; LDH: lactate dehydrogenase; LDL-C: low-density lipoprotein cholesterol; LVEF: left ventricular ejection fraction; MACE: major adverse cardiovascular event; MB: myoglobin; NSTEMI: non-ST segment elevation myocardial infarction; OGTT: oral glucose tolerance test;i; PCI: percutaneous coronary intervention; PTCA: percutaneous transluminal coronary angioplasty; RBC: red blood cells; ROC: receiver operating characteristic; STEMI: ST segment elevation myocardial infarction; TBIL: total bilirubin; TC: cholesterol; TG: triglycerides; TP: total protein; WBC: white blood cell count

Authors' contributions

B-DC, X-CC, SP, Y-TM designed the study; X-CC, Y-NY, X-MG, XM, YH, X-ML, M-TG screened the patients and documented the data. B-DC, X-CC, SP, Y-TM evaluated the literature; B-DC, X-CC, SP, FL undertook the statistical analysis and wrote the first draft of the manuscript. All authors have approved the final manuscript for publication. 


\section{Funding}

This work was supported by grants from National Natural Science Foundation of China (Nos. 81560071), China Postdoctoral Science Foundation (Nos. 2017 M623371), Xinjiang Science and Technology Support Project (Nos. 2016E02073) and Tianshan Innovative Research Team Plan (Nos. 2018D14006)

\section{Availability of data and materials \\ Data and material were available.}

\section{Ethics approval and consent to participate}

The study protocol was approved by the Ethics Committee of First Affiliated Hospital of Xinjiang Medical University.

\section{Consent for publication}

All authors have approved the final manuscript for publication.

\section{Competing interests}

The authors declare that they have no competing interests.

\section{Author details}

${ }^{1}$ Clinical Medical Research Institute of First Affiliated Hospital of Xinjiang Medical University, Urumqi, China. ${ }^{2}$ State Key Laboratory of Pathogenesis, Prevention and Treatment of High Incidence Diseases in Central Asia, Urumqi, China. ${ }^{3}$ Xinjiang Key Laboratory of Cardiovascular Research, Urumqi, Xinjiang, China. ${ }^{4}$ Department of Cardiology, First Affiliated Hospital of Xinjiang Medical University, Urumqi, China. ${ }^{5}$ First Department of Cardiology, People's Hospital of Shaanxi Province, Xi'an, China.

Received: 15 May 2019 Accepted: 30 July 2019

\section{Published online: 07 August 2019}

\section{References}

1. Mandelzweig L, Battler A, Boyko V, Bueno H, Danchin N, Filippatos G, et al. The second euro heart survey on acute coronary syndromes: characteristics, treatment, and outcome of patients with ACS in Europe and the Mediterranean Basin in 2004. Eur Heart J. 2006;27:2285-93.

2. Terkelsen CJ, Lassen JF, Nørgaard BL, Gerdes JC, Jensen T, Gøtzsche LB, et al. Mortality rates in patients with ST-elevation vs. non-STelevation acute myocardial infarction: observations from an unselected cohort. Eur Heart J. 2005:26:18-26.

3. Savonitto S, Ardissino D, Granger CB, Morando G, Prando MD, Mafrici A, et al. Prognostic value of the admission electrocardiogram in acute coronary syndromes. JAMA. 1999;281:707-13.

4. Winter MP, Wiesbauer F, Blessberger H, Pavo N, Sulzgruber P, Huber K, et al. Lipid profile and long-term outcome in premature myocardial infarction. Eur J Clin Investig. 2018:48:e13008.

5. Chakravarty T, Buch MH, Naik H, White AJ, Doctor N, Schapira J, et al. Predictive accuracy of SYNTAX score for predicting long-term outcomes of unprotected left main coronary artery revascularization. Am J Cardiol. 2011; 107:360-6.

6. Serruys PW, Onuma Y, Garg S, Vranckx P, De Bruyne B, Morice MC, et al. 5year clinical outcomes of the ARTS II (arterial revascularization therapies study II) of the sirolimus-eluting stent in the treatment of patients with multivessel de novo coronary artery lesions. J Am Coll Cardiol. 2010;55: 1093-101.

7. Chakrabarti AK, Gibson CM. The SYNTAX score: usefulness, limitations, and future directions. J Invasive Cardiol. 2011:23:511-2.

8. Melina G, Angeloni E, Refice S, Monti F, Serdoz R, Rosato S, et al. Clinical SYNTAX score predicts outcomes of patients undergoing coronary artery bypass grafting. Am Heart J. 2017;188:118-26.

9. Soares AAS, Tavoni TM, de Faria EC, Remalay AT, Maranhão RC, Sposito AC. HDL acceptor capacities for cholesterol efflux from macrophages and lipid transfer are both acutely reduced after myocardial infarction. Clin Chim Acta. 2018:478:51-6

10. Feng L, Yang J, Liu W, Wang Q, Wang H, Shi L, et al. Lipid biomarkers in acute myocardial infarction before and after percutaneous coronary intervention by Lipidomics analysis. Med Sci Monit. 2018;24:4175-82.

11. Michael Gibson C, Korjian S, Tricoci P, Daaboul Y, Yee M, Jain P, et al. Safety and tolerability of CSL112, a reconstituted, infusible, plasma-derived apolipoprotein A-I, after acute myocardial infarction: the AEGIS-I trial (ApoA-I event reducing in ischemic syndromes I). Circulation. 2016:134:1918-30.

12. Rohrer JE, Doganer YC, Merry SP, Angstman KB, Erickson JL, Furst JW. Lowdensity lipoprotein-cholesterol (LDL-C) greater than $100 \mathrm{mg} / \mathrm{dL}$ as a quality indicator: locating risk in person, place and time. J Eval Clin Pract. 2015;21: 735-9.

13. Zhong Z, Liu J, Li B, Li C, Liu Z, Yang M, et al. Serum lipid profiles in patients with acute myocardial infarction in Hakka population in southern China. Lipids Health Dis. 2017:16:246.

14. Lin T, Wang L, Guo J, Liu P, Chen L, Wei M, et al. Association between serum LDL-C and ApoB and SYNTAX score in patients with stable coronary artery disease. Angiology. 2018;69:724-9.

15. Sardella G, Lucisano L, Garbo R, Pennacchi M, Cavallo E, Stio RE, et al. Single-staged compared with multi-staged PCI in multivessel NSTEMI patients: the SMILE trial. J Am Coll Cardiol. 2016;67:264-72.

16. Khan MH, Islam MN, Ahmed MU, Shafique AM, Bari MS, Islam MZ, et al. Comparison between angiographic findings of coronary artery disease in STEMI and NSTEMI patients of Bangladesh. Mymensingh Med J. 2016;25: 221-5.

17. Pan S, Guan GC, LV Y, Liu ZW, Liu FQ, Zhang Y, et al. G-T haplotype established by rs3785889-rs16941382 in GOSR2 gene is associated with coronary artery disease in Chinese Han population. Oncotarget. 2017:8: 82165-73.

18. Athappan G, Patvardhan E, Tuzcu ME, Ellis S, Whitlow P, Kapadia SR. Left main coronary artery stenosis: a meta-analysis of drug-eluting stents versus coronary artery bypass grafting. JACC Cardiovasc Interv. 2013:6:1219-30.

19. Pan S, Liu ZW, Lv Y, Song WQ, Ma X, Guan GC, et al. Association between neutrophilic granulocyte percentage and depression in hospitalized patients with heart failure. BMC Psychiatry. 2016;16:446

20. Pan S, Liu ZW, Shi S, Ma X, Song WQ, Guan GC, et al. Hamilton rating scale for depression-24 (HAM-D24) as a novel predictor for diabetic microvascular complications in type 2 diabetes mellitus patients. Psychiatry Res. 2017;258: 177-83.

21. Krishnaswami A, Maurer MS, Alexander KP. Contextualizing myocardia infarction: comorbidities and priorities in older adults. Am J Med. 2017;130: $1144-7$

22. Garg S, Sarno G, Garcia-Garcia HM, Girasis C, Wykrzykowska J, Dawkins KD, et al. A new tool for the risk stratification of patients with complex coronary artery disease: the clinical SYNTAX score. Circ Cardiovasc Interv. 2010:3:31726.

23. Serruys PW, Onuma Y, Garg S, Sarno G, van den Brand M, Kappetein AP et al. Assessment of the SYNTAX score in the Syntax study. Eurointervention. 2009;5:50-6.

24. Serruys PW, Morice MC, Kappetein AP, Colombo A, Holmes DR, Mack MJ, et al. Percutaneous coronary intervention versus coronary-artery bypass grafting for severe coronary artery disease. N Engl J Med. 2009:360:961-72.

25. Capodanno D, Capranzano P, Di Salvo ME, Caggegi A, Tomasello D Cincotta $G$, et al. Usefulness of SYNTAX score to select patients with left main coronary artery disease to be treated with coronary artery bypass graft. JACC Cardiovasc Interv. 2009:2:731-8.

26. Sianos G, Morel MA, Kappetein AP, Morice MC, Colombo A, Dawkins K, et al. The SYNTAX score: an angiographic tool grading the complexity of coronary artery disease. Eurointervention. 2005;1:219-27.

27. Pamir N, Hutchins PM, Ronsein GE, Wei H, Tang C, Das R, et al. Plasminogen promotes cholesterol efflux by the ABCA1 pathway. JCI Insight. 2017:2:92176.

28. Tricoci P, D'Andrea DM, Gurbel PA, Yao Z, Cuchel M, Winston B, et al. Infusion of reconstituted high-density lipoprotein, CSL112, in patients with atherosclerosis: safety and pharmacokinetic results from a phase $2 a$ randomized clinical trial. J Am Heart Assoc. 2015:4:e002171.

29. Aksakal E, Tanboga IH, Kurt M. Predictors of coronarylesions complexity in patients with stable coronary artery disease. Angiology. 2013:64:304-9.

30. de Lemos JA, Morrow DA, Gibson CM, Murphy SA, Sabatine MS, Rifai N, et al. The prognostic value of serum myoglobin in patients with non-STsegment elevation acute coronary syndromes. Results from the TIMI 11B and TACTICS-TIMI 18 studies. J Am Coll Cardiol. 2002:40:238-44

\section{Publisher's Note}

Springer Nature remains neutral with regard to jurisdictional claims in published maps and institutional affiliations. 